\title{
Experimental Study on Influences of Surface Materials on Cavitation Flow Around Hydrofoils
}

\author{
Jiafeng Hao®i, Mindi Zhang* and Xu Huang
}

\begin{abstract}
In order to resist on the cavitation erosion, many researchers try to change the solidity and tenacity of the coatings, but ignore the influence of surface characteristics of materials on cavitation flow and the interaction with each other. In this paper, high speed visualization system is used to observe the cavitation flow patterns in different stage. After comparing the characteristics of cavitation flow around hydrofoils made of aluminum (Foil A), stainless steel (Foil B) and the hydrofoil painted with epoxy coating (Foil C), the study shows that material has a significant effect on the cavitation flow. Firstly, when the incipient cavitation occurs, cavitation number of Foil A is highest among three hydrofoils, generating horseshoe vortex randomly. For Foil B and Foil C, it shows in the form of free bubbles. When the sheet cavitation occurs, Foil A has the highest cavitation number and shortest period, which is contrary to Foil C. And cavity consists of lots of small finger-like cavities. For Foil B and Foil C, it both constitutes with many bubbles. Compared with the high-density and small-scale cavities over surface of Foil C, the cavity of Foil B has larger scale and less density, which causes a minimal scope of influence of the re-entrant jet and strong randomness. When the cloud cavitation occurs, Foil $\mathrm{C}$ has the lowest cavitation number and shortest period. Secondly, compared with aluminum, both of stainless steel and epoxy coating restrains the occurrence and development of cavitation, and stainless steel and epoxy coating performs better than aluminum. For inception and sheet cavitation, stainless steel performs better than epoxy coating and aluminum. For cloud cavitation, epoxy coating performs better than stainless steel and aluminum. The objective of this paper is applied experimental method to investigate the effect of surface materials on cavitation around Clark-Y hydrofoils.
\end{abstract}

Keywords: Cavitation, Coating, Hydrofoil, Horseshoe vortex, Experiment

\section{Introduction}

Cavitation occurs when pressure within the fluid drops below the saturated vapor pressure, which is closely related to the local temperature and flow structure. Consequently, the negative pressures are relieved by means of forming gas filled or gas and vapor filled cavities [1]. Those cavities will transport with the flow to downstream and when the local pressure outside the cavities rise back to higher than the vapor pressure, the cavities will be compressed and finally collapse. If this process just happens in the vicinity of the solid surface, a high speed micro-jet towards the solid surface will generate and attack the surface inducing cavitation erosion [2].

*Correspondence: zmd1971@bit.edu.cn

School of Mechanical Engineering, Beijing Institute of Technology, Beijing 100081, China
In order to resist on the cavitation erosion, many researchers make use of different kinds of coatings and try to change the solidity and tenacity of the materials by many methods, Feng et al. [3] explored the cavitation erosion resistance of the MAO coating, cavitation tests were performed by using a rotating-disk test rig. Espitia et al. [4] evaluated cavitation erosion resistance of low temperature plasma nitrided martensitic stainless steel. Franc et al. [5] investigated three different materials: an Aluminum alloy, a Nickel Aluminum Bronze alloy and a Duplex Stainless Steel via pitting tests, the conclusion indicated that the damage is not correlated in simple terms with the elastic limit determined from conventional tensile tests and it is conjectured that other parameters, such as the strain rate might play a significant role. Laguna-Camacho et al. [6] conducted cavitation erosion tests on different materials, such as pure aluminium 
and mild steel evaluate the performance of all the tested materials at different testing conditions. Boinovich et al. [7] presented a modification of a stainless steel surface to impart super hydrophobic properties to it that were robust with respect to mechanical stresses associated with cyclic icing/deicing treatment, as well as to longterm contact with aqueous media and high humidity. Li et al. [8] studied that Ti-6Al-4V alloy was processed by pack carburizing to improve the cavitation erosion behavior. Zavareh et al. [9] investigated the tribological properties of the plasma-sprayed $\mathrm{Al}_{2} \mathrm{O}_{3}-40 \mathrm{wt} \% \mathrm{TiO}_{2}$ coating with a tribometer (pin-on-disc) to evaluate and compare the wear properties of coated and uncoated samples under different loads. Aperador et al. [10] researched the improving the erosion corrosion resistance cavitation of coatings TiN on aluminum exposed to mixtures bioethanol. Qiu et al. [11] researched on cavitation erosion and wear resistance performance of coatings, results showed that excellent wear-resisting performance of ceramic coatings, which is better than wear-resistance of stainless steel, cast iron and high chrome alloy steel. But the excellent wear-resisting performance could not guarantee a good erosion-resisting performance.

However, the influence of surface characteristics of materials on cavitation flow and the interaction with each other are ignored. Some researchers [12-15] have noted the phenomenon and started to work on them. Hajian et al. [16] found that the improvement in cavitation erosion resistance is attributed to smaller grain structure, lower fraction of twin boundaries, and favorable crystallographic orientation of grains in FSP samples. Chi et al. [17] found that the cavitation erosion resistance was greater in coating materials with better ductile and tough properties than in coating materials with higher strength or hardness. And some researchers focus on the relationship between materials and single bubble collapse forming from the cavitation flow. Choi et al. [18] examines a numerical fluid-material interaction approach to investigate this relationship between material pitting and cavitation field impulsive pressures. Some numerical simulations are conducted to investigate pitting formation from the combined bubble dynamics and material mechanics viewpoints $[15,19-23]$.

It is known that cavitation often involves complex interactions between turbulent flow dynamics with large variations in fluid density and pressure fluctuations [24-29] and surface materials. Therefore, it's necessary to research the interactions between bubbles clusters dynamics in cavitation flow and the surface.

The objective of the present work is to investigate systematically the influences of surface materials on cavitation flow.

\section{Experimental Set-up}

In present paper, tests are conducted in a high speed water tunnel of Beijing Institute of Technology, the sketch map of the tunnel is shown in Figure 1. The primary characteristics of experimental set-up are summarized here, and more information about the instrumentation with experimental uncertainties and sketch map of foil's position in the test section is available in Ref. [30]. In the experiments, the development of the cavitating flow around hydrofoils are observed for various cavitation numbers at velocity of $V_{\infty}=8 \mathrm{~m} / \mathrm{s}$. Figure 2 shows the effect drawing and sketch map of foil's arrangement, the chord length of the hydrofoils is $70 \mathrm{~mm}$, and the angle of attack is set as $8^{\circ}$.

Three hydrofoils are machined from the same surface roughness $\left(R_{a}=2 \mu \mathrm{m}\right)$ and different material. As shown in Figure 3, Foil A is made of aluminum, Foil B and Foil C is made of stainless steel, but Foil $\mathrm{C}$ is painted with epoxy coating, the thickness is $0.5 \mathrm{~mm}$.

During the process of experiment, we can adjust speed of the axial-flow pump to control the inlet velocity, or control the ambient pressure to adjust cavitation number. Furthermore, the series of pictures of the experiment can be obtained with the help of high-speed cameras, and analyze the structure of cavitation, the law of the cavitation development and flow visualization in detail.

The cavitation number is a dimensionless parameter describing the cavitation state, which is always represented by $\sigma$ and defined as follows:

$$
\sigma=\frac{P_{\infty}-P_{v}}{0.5 \rho V_{\infty}^{2}}
$$

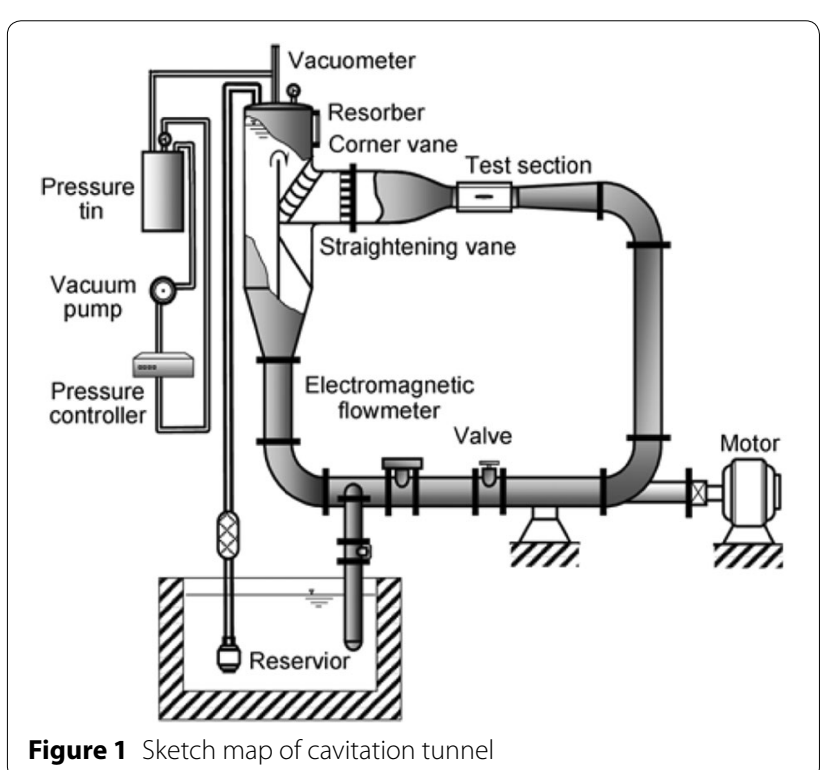




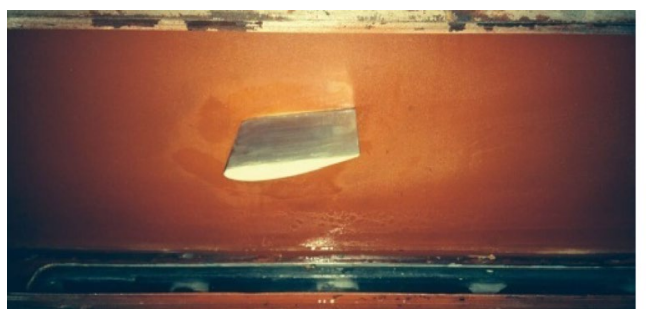

a Effect drawing of foil's arrangement

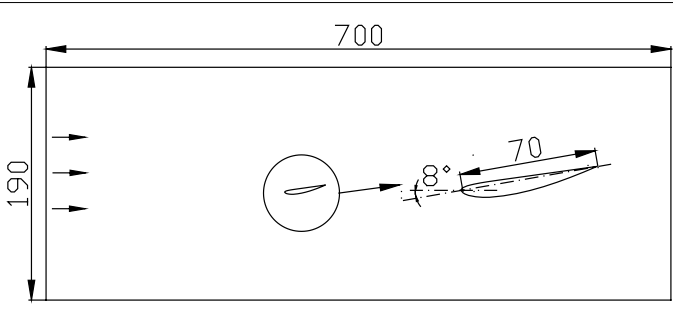

b Sketch map of foil's arrangement

Figure 2 Effect drawing and sketch map of foil's arrangement

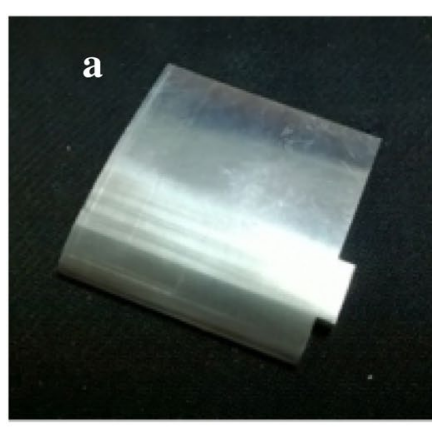

a Foil A

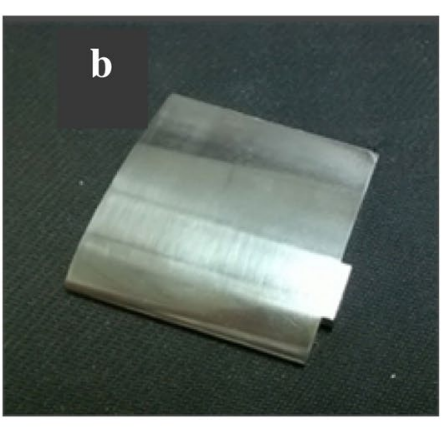

b Foil B

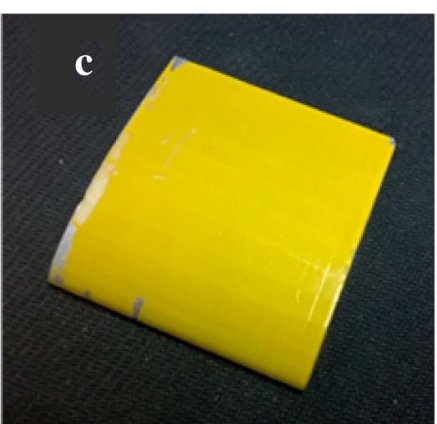

c Foil C

Figure 3 Photos of hydrofoils made of different materials

The Reynolds number is defined as follows, in this experiment, the Reynolds number is $5.6 \times 10^{5}$ :

$$
R e=\frac{V_{\infty} l}{v} .
$$

The location coefficient $x^{\prime}$ and $y^{\prime}$ are defined as follows:

$$
x^{\prime}=\frac{x}{l \cos \alpha}, \quad y^{\prime}=\frac{y}{l \cos \alpha} .
$$

The time coefficient is defined as follows:

$$
t^{\prime}=\frac{t-t_{0}}{T} .
$$

Here, $P_{\infty}, P_{v}$ and $V_{\infty}$ are upstream pressure, vapor pressure and velocity at free stream respectively. $\rho$ is the water density at the room temperature. $v, l, \alpha$ are kinetic viscosity, chord length of hydrofoil, the angle of attack, respectively. $x$ and $y$ are horizontal and vertical position coordinates in the upward view. $t_{0}$ is initial moment, $T$ is the period of the cavitation flow.

\section{Results and Discussion}

During the process of experiment, the high speed visualization system is adopted to observe the patterns and development of the cavitation flow around the hydrofoils made of different materials. The ambient pressure is adjusted to satisfy conditions for different cavitation number.

\subsection{Results Analysis for Inception and Sheet Cavitation}

Figure 4 shows the upward view of inception and sheet cavitation around Foil A, Foil B and Foil C for different cavitation number. It can be found that no cavitation occurs in the flow fields around Foil A, Foil B and Foil C when $\sigma$ is 1.96 . When $\sigma$ is 1.88 , the incipient cavitation occurs around Foil $\mathrm{A}$, which generates horseshoe vortex randomly. When $\sigma$ is 1.82 , the incipient cavitation occurs around Foil C. At $x^{\prime}=0.1$, some free bubbles appear in the leading edge of surface of the Foil $\mathrm{C}$, and the bubbles are neat relatively and linear nearly. The flow field of Foil $B$ is still in the stage of no cavitation. Attached spot cavitation has already occurred around Foil $\mathrm{A}$ at this time.

When $\sigma$ is 1.65 , cavity in the surface of Foil A develops to the entire spanwise width, the tail of cavitation develops to $x^{\prime}=0.5$. Inception cavitation occurs on the suction surface of the Foil $B$, and the free random bubbles with different sizes are commonly observed, which have big differences with the flow patterns of the inception cavitation around Foil $\mathrm{C}$ when $\sigma$ is 1.82 . Cavitation flow around Foil $\mathrm{C}$ develops into attached sheet cavitation. The leading edge cavity develops to $x^{\prime}=0.05$, the cavity 


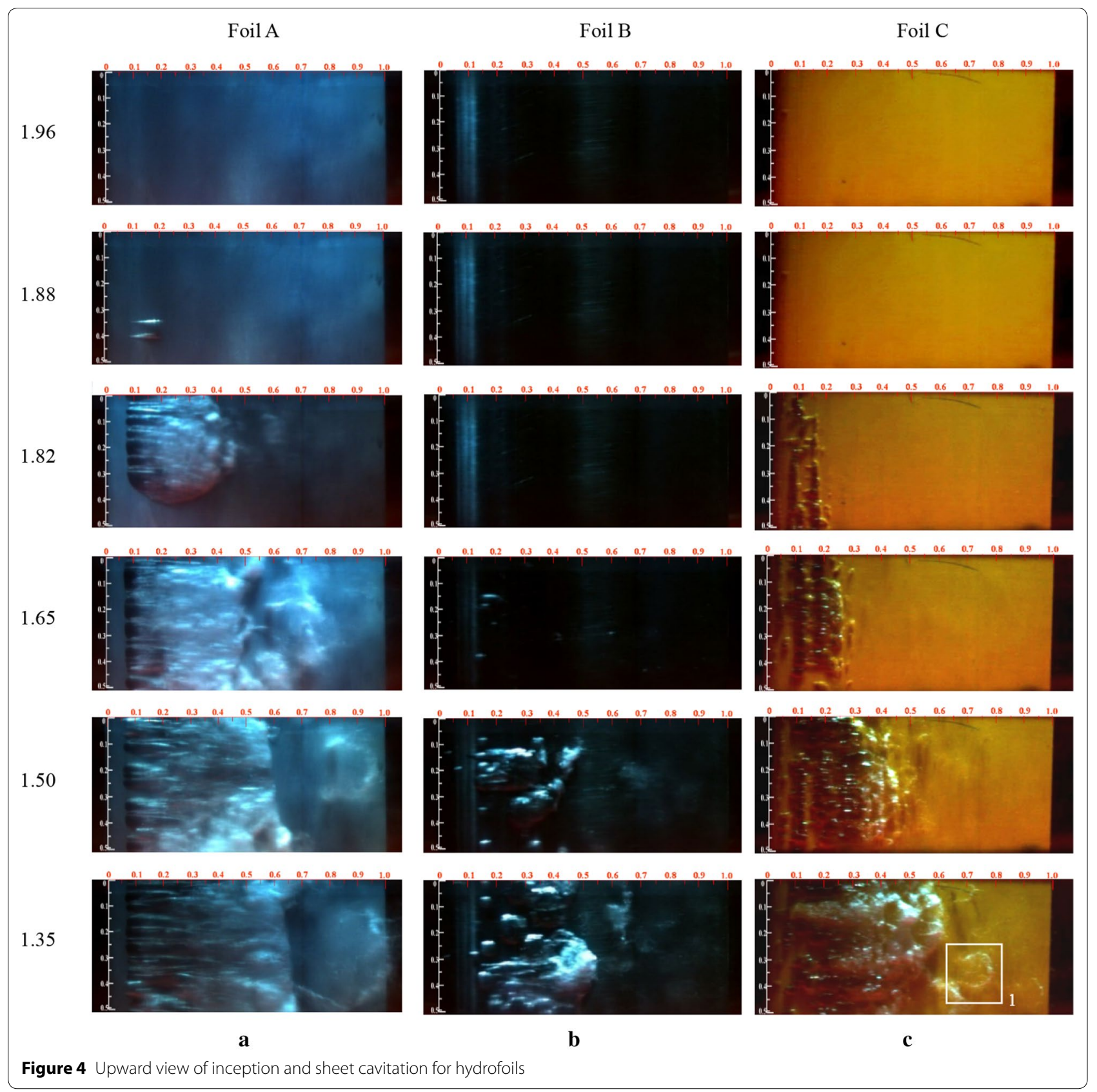

length increased to $0.2 \mathrm{C}$, and the bubbles scale increase to $1-1.5 \mathrm{~mm}$.

Cavitation flow around Foil A, Foil B, Foil C all develops into attached sheet cavitation when $\sigma$ is 1.50. Cavitation flow around Foil $\mathrm{A}$ is the most intense, and is certain periodic. Sheet cavitation around Foil B is consisted of two parts. One part of the cavitation is free bubbles at $y^{\prime}=0.33$ and $y^{\prime}=0.46$; another part is attached sheet cavitation at $y^{\prime}=0.13-0.26$, which shows that the cavitation is unsteady. The cavitation around Foil $\mathrm{C}$ presents as many discontinuous bubbles. After moving along the surface of the foil to $x^{\prime}=0.35$, transparent bubbles collapse into lots of small ones, and form white bubbles cluster.

The length of the cavity in the surface of the Foil A increases, and the longest can be up to $0.65 \mathrm{C}$ in the fluctuation process. The flow field of Foil B is still in the unsteady state of combination of a single cavity and attached sheet cavitation. The bubbles' size increases owing to the pressure distribution of the suction side. The diameter of the bubble is $1.5 \mathrm{~mm}$ at $x^{\prime}=0.12, y^{\prime}=0.27$, 
diameter of the bubble is $3 \mathrm{~mm}$ at $x^{\prime}=0.17, y^{\prime}=0.25$, and diameter of the bubble is $6 \mathrm{~mm}$ at $x^{\prime}=0.25, y^{\prime}=0.16$. The cavity length in the surface of Foil $\mathrm{C}$ increase to $0.5 \mathrm{C}$ when $\sigma$ is 1.35 , the bubbles moving to $x^{\prime}=0.5$ collapse into lots of small ones, and some form horseshoe vortex as shown box 1 in Figure 4. Compared with cavitation flow around Foil A and Foil B, it is difficult to form a stable attached cavity in the surface of hydrofoil, and growth of a single cavity is restricted. As the density of bubbles decreases, it consists of part of attached cavity and free bubbles, and the diameter of the single bubbles increases to $6 \mathrm{~mm}$. When the density of bubbles is close to zero, typical attached sheet cavitation around hydrofoil occurs as shown in Figure 4(a).

Figures 5, 6 show upward view of the time evolution of sheet cavitation around hydrofoils. We have presented in Figure 5(a), the period of sheet cavitation around Foil A is about $12.6 \mathrm{~ms}$ when $\sigma$ is 1.50 , and in Figure 5(b) it is about $14.3 \mathrm{~ms}$ for Foil $\mathrm{B}$. The density of bubbles in the surface of Foil B increases, and the bubbles appear at $x^{\prime}=0.05$. The bubbles along the surface keep growing to relatively ones, and then breaks into small bubbles cluster when the diameter increases to $6 \mathrm{~mm}$ at $x^{\prime}=0.4$. Collapse direction is from back to the front, and in contrast to the flow. During this process, re-entrant jet forming in the trailing edge of cavity for Foil B is extremely unsteady. Shown as Figure 6, the process of the sheet cavitation around Foil $\mathrm{C}$ contains two parts when $\sigma$ is 1.35: the development of cavity, the shedding and collapse of cavity result from development of re-entrant jet.

Shown as Figures 5, 6, re-entrant jet push the flow towards the leading edge at $x^{\prime}=0.15$ when $\sigma$ is 1.5 . For Foil C, re-entrant jet can reach to $x^{\prime}=0.25$, and for Foil $\mathrm{B}$, it can only reach to $x^{\prime}=0.42$. Figure 7 shows the sketch map of sheet cavitation around the hydrofoils. Sheet cavitation around Foil A consists of lots of small cavities in form of finger-like ones as shown in Figure 7(a). The closed region in the trailing edge shows a continuous curve profile, then cavities shed and collapse into a large number of finely ones. Shown as Figure 7(b) and Figure $7(\mathrm{c})$, low pressure region owing to collapse of single bubble in the surface of Foil B results in two-phase mixture flow moving to the front. Because of influences of density of bubbles and position of collapse, it is a greater randomness to form re-entrant jet. Meanwhile, lower density of bubbles and higher content of liquid result in re-entrant jet owing to collapse of bubbles dissipates quickly, the scope of influence becomes smaller, and the rear part of cavity is very irregular. As shown in Figure $7(\mathrm{~d})$, the cavity around Foil $\mathrm{C}$ consists of highdensity small bubbles, the region of movement of bubbles covered the suction side of Foil $C$, thus, re-entrant jet forming in the surface is steady and continuous relatively, which result in the neat fracture and shedding of cavity. So, there is a great influence of cavity patterns on forming and moving of re-entrant jet.

\subsection{Results Analysis for Cloud Cavitation}

Shown as Figure 8, cloud cavitation around Foil A occurs in form of finger-like ones when $\sigma$ is 1.02 as shown in Figure 8 , and the period of the cavitation is $52 \mathrm{~ms}$. According to the development of cavity, the process of the cloud cavitation contains two stages: (1) when $t=0-36 \mathrm{~ms}$, attached cavity develops the along the surface of Foil A toward the trailing edge, and reach to the trailing edge when $t=36 \mathrm{~ms}$; (2) when $t=36-52 \mathrm{~ms}$, the re-entrant jet owing to the adverse pressure gradient moves along the suction side toward the leading edge, leading to fluctuation and shedding of cavity toward the front, and forming shedding of bubbles cluster and collapse in the end of period.

Figure 9 shows the pattern of the cavitation flow field around Foil B. The process of the cloud cavitation contains two stages, (1) when $t=0-34 \mathrm{~ms}$, attached cavity develops along the surface of Foil A toward the trailing edge, and reaches to the trailing edge when $t=34 \mathrm{~ms}$. (2) when $t=34-52.8 \mathrm{~ms}$, the re-entrant jet owing to the adverse pressure gradient moves along the suction side toward the leading edge, leads to fluctuation and shedding of cavity toward the front, and forms shedding and collapsing of bubbles cluster in the end of period. Similar to sheet cavitation around Foil B as shown in Figure 9, cloud cavitation around Foil B consists of two parts. The front part of cavity is transparent bubbles, and the rear part of cavity is small bubbles cluster owing to collapse of bubbles. The pictures from $t=0$ to $t=18 \mathrm{~ms}$ show that the trailing edge of cavity develops from $x^{\prime}=0.35$ to $x^{\prime}=0.55$. In this position, cavity collapses into small bubbles cluster and develops toward the rear, which results from the pressure distribution of the suction side. The length of cavity reaches to $x^{\prime}=1.0$ when $t=34 \mathrm{~ms}$, and start to dissipate later. Owing to development of reentrant jet, cavity shrinks to $x^{\prime}=0.4$ when $t=52.8 \mathrm{~ms}$, and the period is over.

Shown as Figure 10, the process of the cloud cavitation contains two stages: (1) when $t=0-32 \mathrm{~ms}$, attached cavity develops toward the trailing edge along the surface of Foil $C$, and reach to the trailing edge when $t=32 \mathrm{~ms}$. (2) When $t=32-50 \mathrm{~ms}$, cavity in the surface of Foil $\mathrm{C}$ fluctuates, sheds and collapses from the rear region to the front. At $t=38 \mathrm{~ms}$, cavity sheds completely, forming shedding of bubbles cluster and then collapse in the end of period. Subsequently, attached cavity in the front of suction side sheds again, and forms several obvious vortexes. 
Upward view
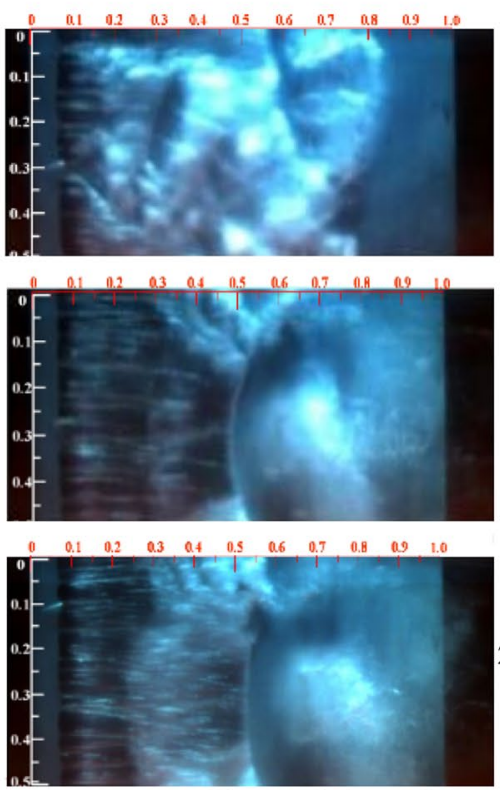

a Time evolutions of sheet cavitation around Foil $\mathrm{A}(\sigma=1.50)$

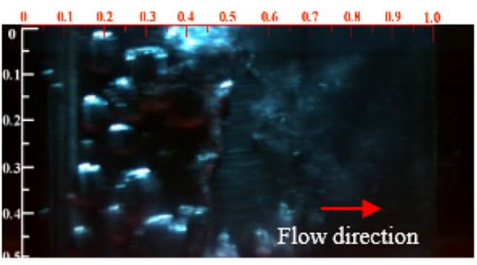

$8 \mathrm{~ms}$

$10 \mathrm{~ms}$

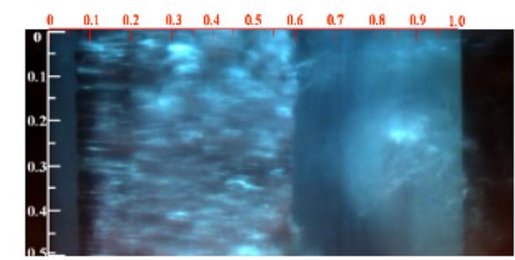

$2.6 \mathrm{~ms}$
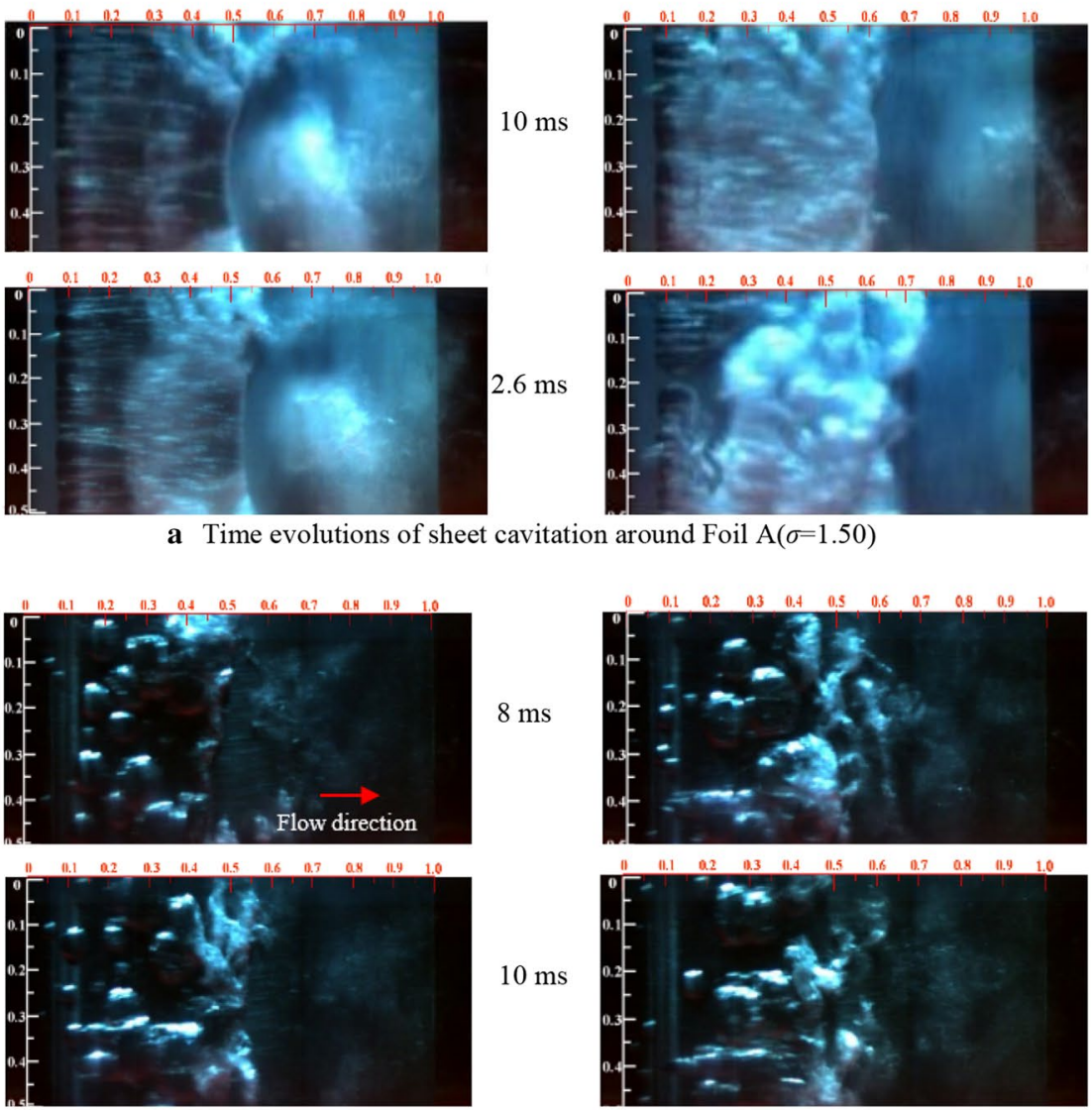

$10 \mathrm{~ms}$

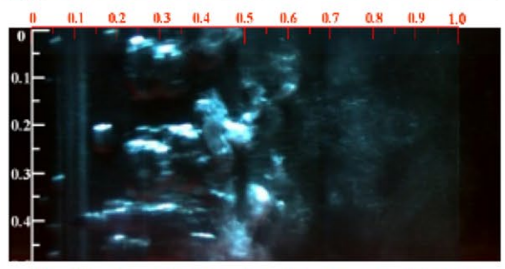

$12 \mathrm{~ms}$
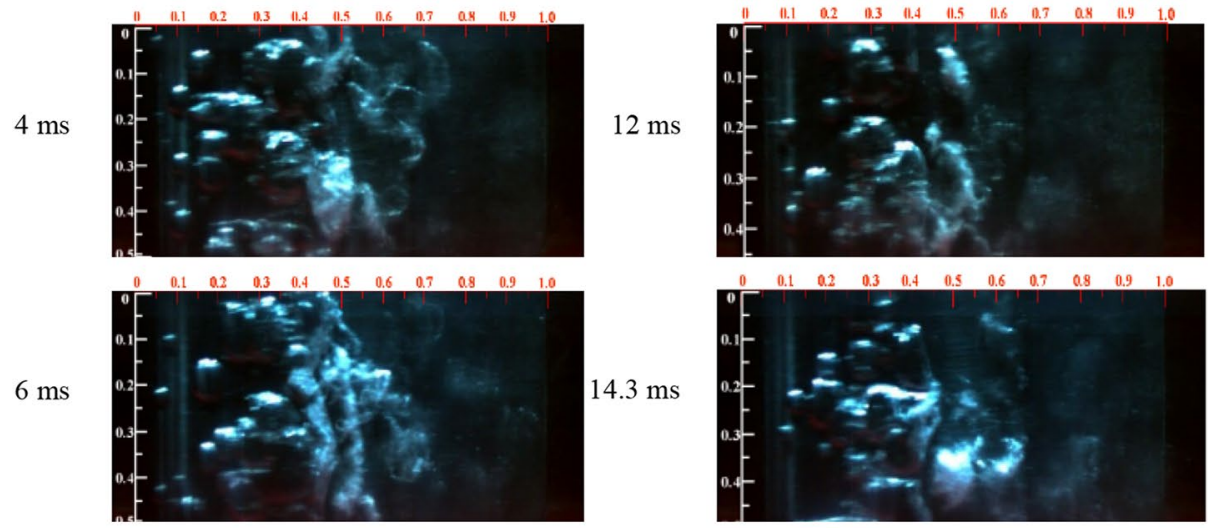

$14.3 \mathrm{~ms}$

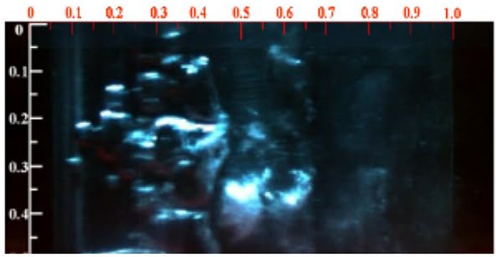

b Time evolutions of sheet cavitation around Foil $\mathrm{B}(\sigma=1.35)$

Figure $\mathbf{5}$ Time evolutions of sheet cavitation 


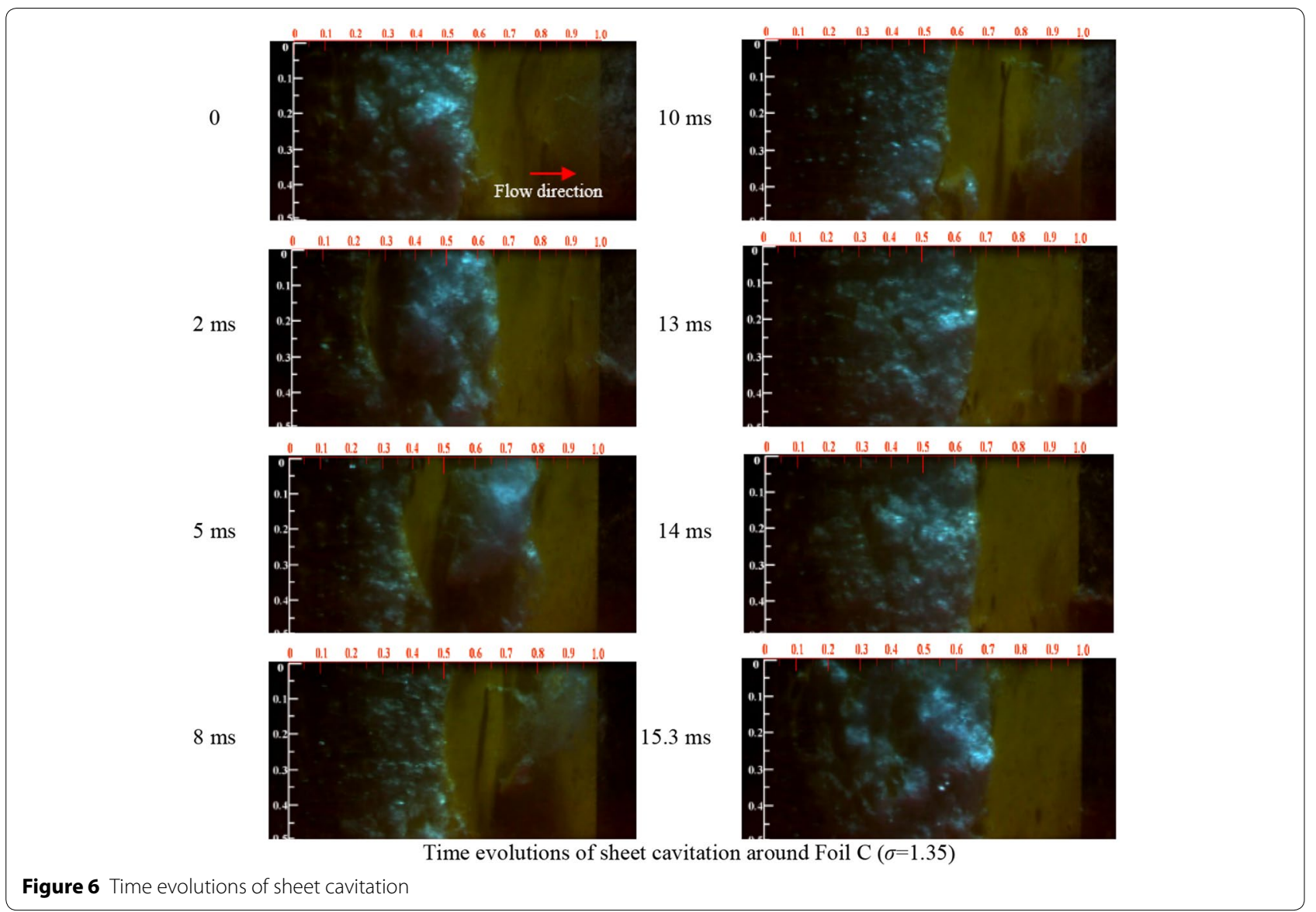

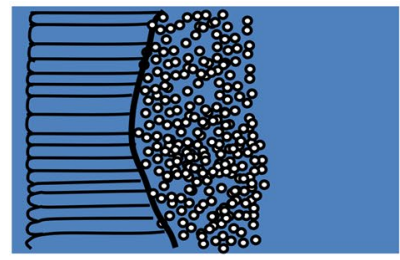

a Upward view of Foil A

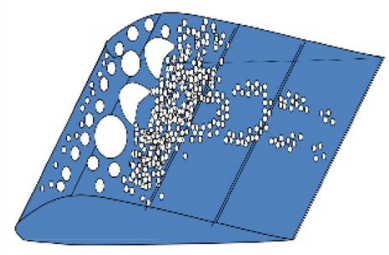

b Side view of Foil B

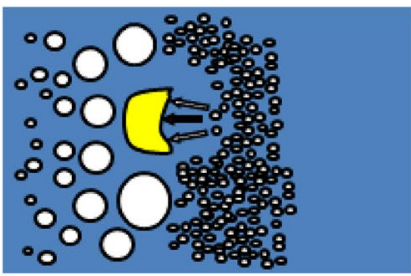

c Upward view of Foil B

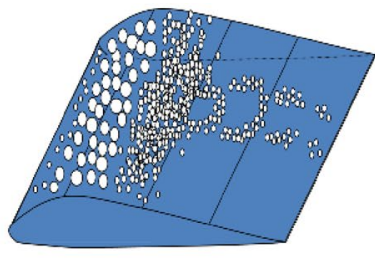

d Side view of Foil C

Figure 7 Sketch map of sheet cavitation around the hydrofoils

After comparing with each other, we find that the period and process of cloud cavitation around Foil A, Foil $\mathrm{B}$ and Foil $\mathrm{C}$ are all different, which indicates that surface materials influence cavitation flow around hydrofoils. Materials influence the pattern of the cavitation flow field around hydrofoils by forming a large number of free bubbles. The attached cavity consists of the free bubbles and water in the flow field, but its structure is different from typical attached cavity. The bubbles cluster consisting of two-phase mixture influences development of the re-entrant jet toward the front, and then influences the period and process of cavitation flow. In summary, materials influence the pattern of the cavitation, and then influence the structure of cavitation and development of re-entrant jet, which leads to the differences of cavitation flow around hydrofoil made of different material.

Materials influence the cavitation flow, but it still can be divided into two typical stages. Growth of attached cavity moves along the surface of hydrofoil and development of re-entrant jet result in the shedding and collapsing of cavity to forming bubbles cluster. 
Upward view
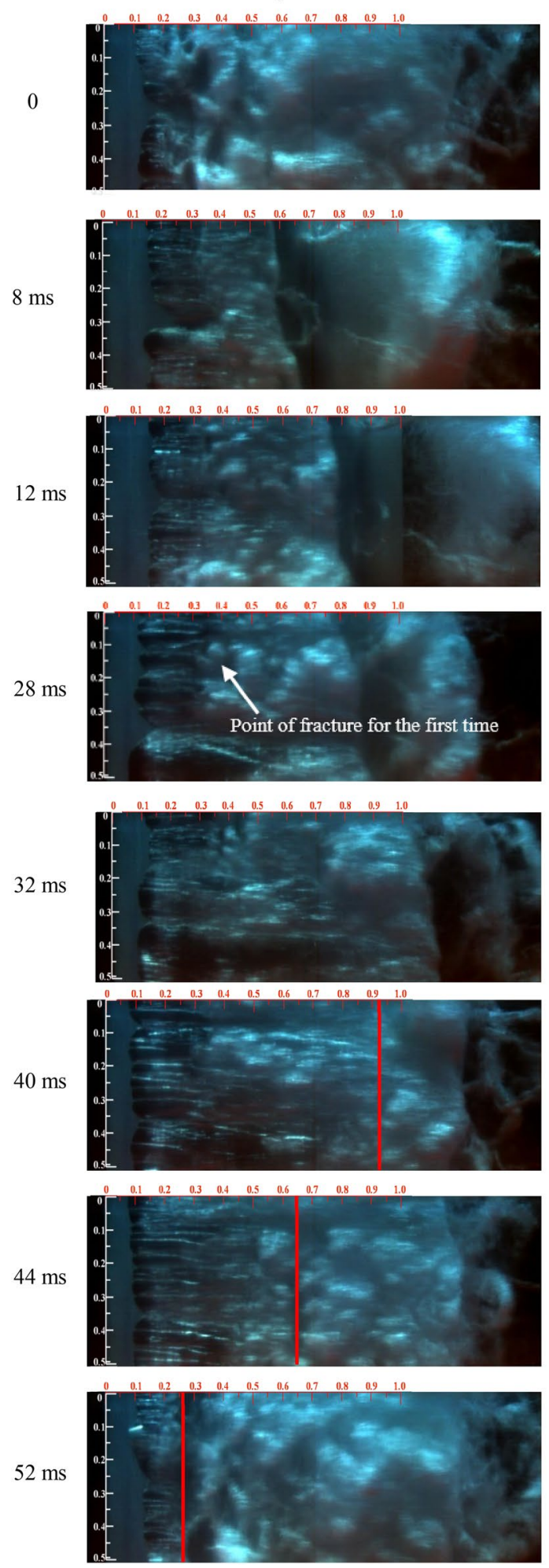

Side view
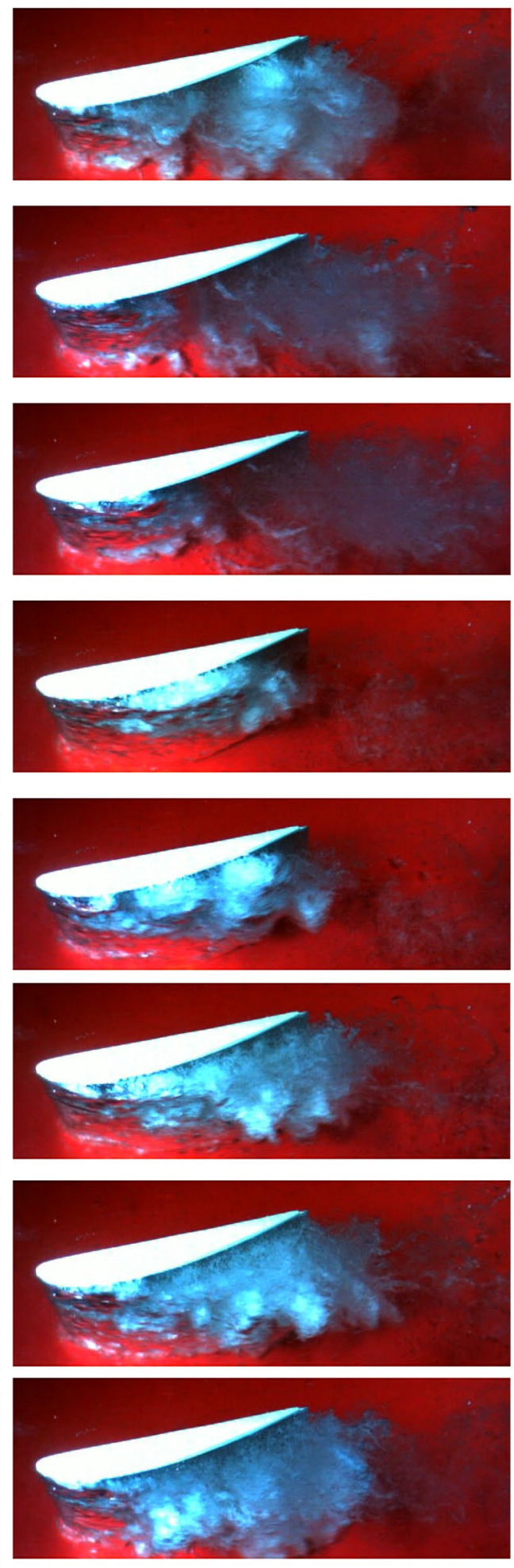

Figure 8 Time evolutions of cloud cavitation around Foil A $(\sigma=1.02)$ 


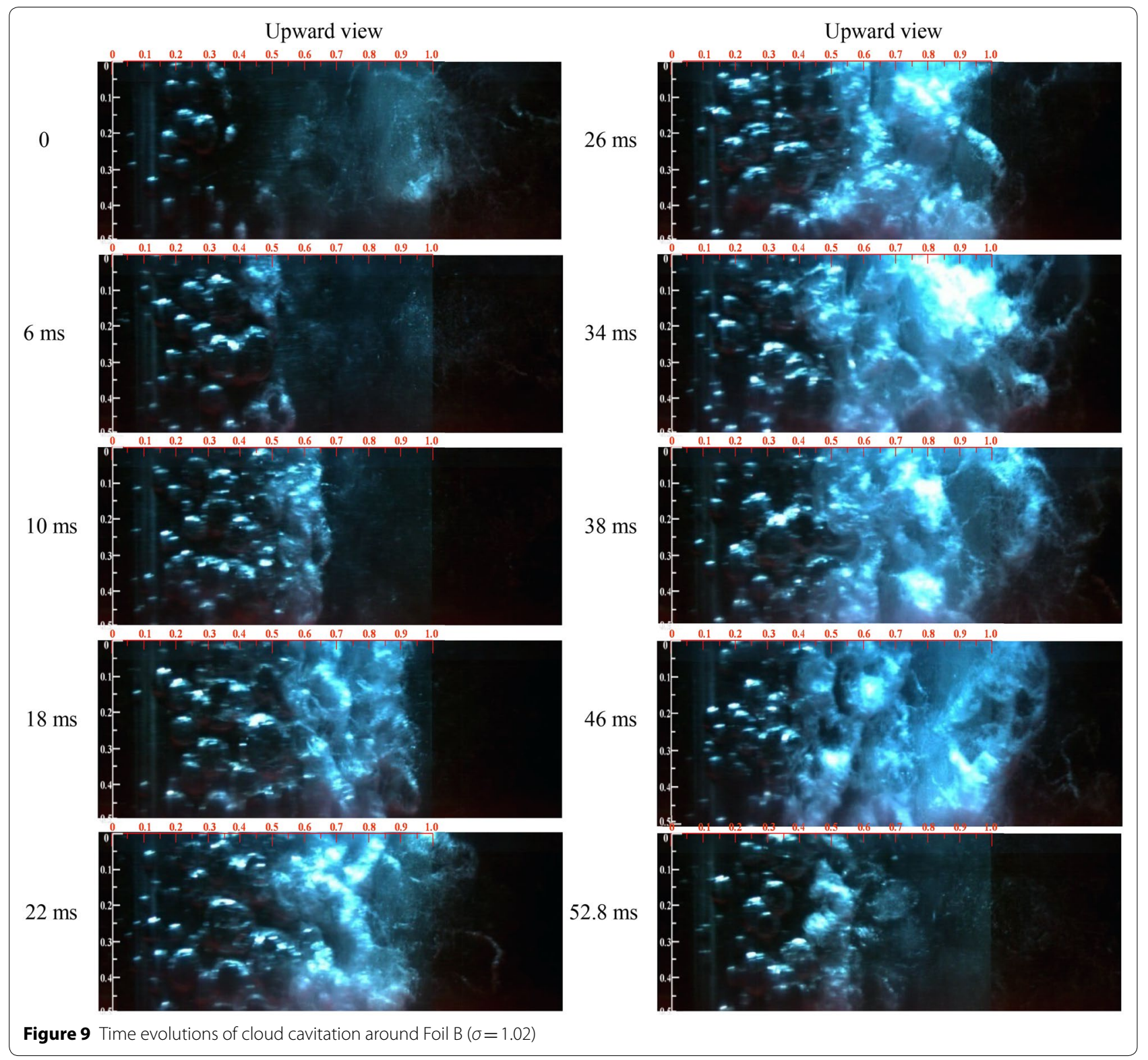

\section{Conclusions}

Influences of several surface materials on cavitation flow around hydrofoils are studied experimentally. High speed visualization system is executed to observe the cavitation flow patterns and structure in different stage. The study shows that material influences significantly the cavitation flow. The main results are as follows.

1) Material influence on the development of cavitation flow, which still consists of incipient cavitation, sheet cavitation and cloud cavitation.

2) Material takes a certain effect on the incipient cavitation flow over the hydrofoil. When $\sigma$ is 1.88 , the incipient cavitation occurs around Foil A, and generates horseshoe vortex, randomly. When $\sigma$ is 1.65 , the incipient cavitation occurs around Foil B. Incipient cavitation occurs around Foil $\mathrm{C}$ with $\sigma$ of 1.82. Incipient cavitation around Foil B and Foil $\mathrm{C}$ appear as free bubbles, and the bubbles of Foil $\mathrm{C}$ are neat relatively.

3) Material takes a certain effect on the sheet cavitation flow over the hydrofoil. When $\sigma$ is 1.50 , cavitation flow around Foil A develops into attached sheet cavitation, whose period is $12.6 \mathrm{~ms}$. The cavity consists of lots of small cavities in form of finger-like ones. When $\sigma$ is 1.35 , cavitating flows around Foil B and Foil $\mathrm{C}$ develop into attached sheet cavitation. The 


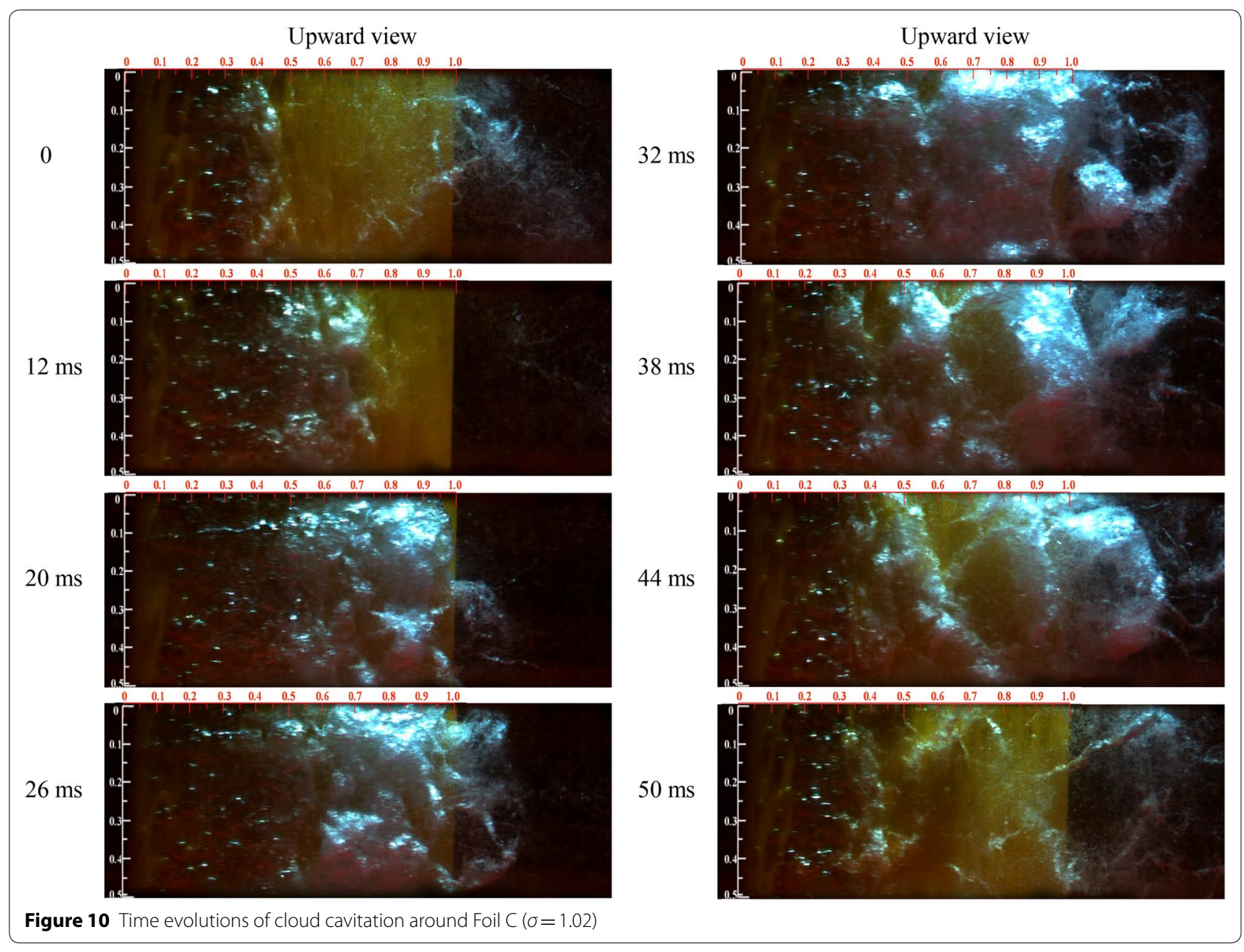

period of Foil B is $14.3 \mathrm{~ms}$, and the period of Foil $\mathrm{C}$ is $15.3 \mathrm{~ms}$. The sheet cavitation around Foil Band Foil C are both constituted with many bubbles. Compared with the high-density and small-scale cavities in the surface of Foil C, the cavity on the surface of Foil B has larger scale and less density, which causes a minimal scope of influence of the re-entrant jet at Foil B tail and its strong randomness.

4) Material takes a great effect on the cloud cavitation flow over the hydrofoil. Cavitating flows around Foil $\mathrm{A}$ and Foil B develop into cloud cavitation when $\sigma$ is 1.02, while cavitation flow around Foil $\mathrm{C}$ develops into cloud cavitation with $\sigma$ of 0.87 . The periods of Foil A, Foil B and Foil C are $52 \mathrm{~ms}, 52.8 \mathrm{~ms}$ and $50 \mathrm{~ms}$, respectively. Cloud cavitation around three kinds of hydrofoils can be divided into two stages. One is the growth and accumulation of cavity near the trailing region of hydrofoils, and the other is off-breaking of the cavity and its cloud dissipation caused by formation of the re-entrant jet.
5) Compared with the cavitation around Foil A made of aluminum, both of Foil B made of stainless steel and Foil $\mathrm{C}$ painted with epoxy coating restrains the occurrence and development of cavitation, and stainless steel and epoxy coating performs better than aluminum. For The inception and sheet cavitation, stainless steel performs better than epoxy coating and aluminum. And for cloud cavitation, epoxy coating performs better than stainless steel and aluminum

\section{Authors' Contributions}

$\mathrm{MZ}$ was in charge of the whole trial; $\mathrm{JH}, \mathrm{XH}$ and $\mathrm{MZ}$ wrote the manuscript; $\mathrm{JH}$, $\mathrm{XH}$ and $\mathrm{MZ}$ assisted with sampling and laboratory analyses. All authors read and approved the final manuscript.

\section{Authors' Information}

Jiafeng Hao, born in 1992, is currently a master candidate at School of Mechanical Engineering, Beijing Institute of Technology, China. His research interests include cavitation and fluid mechanics. 
Mindi Zhang, born in 1971, is currently an associate professor at Beijing Institute of Technology, China. She received his master degree on mechanical engineering from Tianjin University, China, in 2000.

Xu Huang, born in 1990, is a master candidate at School of Mechanical Engineering, Beijing Institute of Technology, China. His research interests include cavitation and fluid mechanics.

\section{Competing Interests}

The authors declare no competing financial interests.

\section{Funding}

Supported by National Natural Science Foundation of China (Grant No.

51106009).

Received: 11 April 2017 Accepted: 18 April 2019

Published online: 09 May 2019

\section{References}

[1] G K Batchelor. An introduction to fluid dynamics. New York: Cambridge University Press, 1967.

[2] D H Trevena. Cavitation and tension in liquids. IOP Publishing Ltd., Great Britain, 1987.

[3] C Feng, S Jiang, J Liang. Cavitation erosion resistance of microarc oxidation coating on aluminium alloy. Applied Surface Science, 2013, 280(8): 287-296.

[4] L A Espitia, L Varela, C E Pinedo, et al. Cavitation erosion resistance of low temperature plasma nitrided martensitic stainless steel. Wear, 2013, 301(s1-2): 449-456.

[5] J P Franc, M Riondet, A Karimi, et al. Material and velocity effects on cavitation erosion pitting. Wear, 2012, s 274-275(275): 248-259.

[6] J R Laguna-Camacho, R Lewis, M Vite-Torres, et al. A study of cavitation erosion on engineering materials. Wear, 2013, 301(1-2): 467-476.

[7] B Boinovich, A M Emelyanenko, V K Ivanov, et al. Durable icephobic coating for stainless steel. Acs Applied Materials \& Interfaces, 2013, 5(7): 2549-2554.

[8] H Li, Z Cui, Z Li, S Zhu, et al. Microstructure and cavitation erosion properties of ceramic coatings fabricated on Ti-6Al-4V alloy by pack carburizing. Journal of Materials Engineering and Performance, 2014, 23(8): 2772-2779.

[9] M A Zavareh, AADM Sarhan, BBA Razak, et al. Plasma thermal spray of ceramic oxide coating on carbon steel with enhanced wear and corrosion resistance for oil and gas applications. Ceramics International, 2014, 40(9): 14267-14277.

[10] W Aperador, E Ruíz, A Delgado. Improving the erosion corrosion resistance cavitation of coatings TiN on aluminum exposed to mixtures bioetanol. International Journal of Electrochemical Science, 2014, 9(12): 7277-7286.

[11] N Qiu, L Wang, S Wu, et al. Research on cavitation erosion and wear resistance performance of coatings. Engineering Failure Analysis, 2015, 55: 208-223.
[12] M Williams, E Kawakami, E Amromin, et al. Effects of surface characteristics on hydrofoil cavitation. Proc. of the 7th Int. Symp. on Cavitation, USA, 2001: 1-6.

[13] L Zhang, V Belove, H Q Wang, et al. Controlled cavitation at nanol microparticle surfaces. Chemistry of Materials, 2014, 26: 2244-2248.

[14] S Lu, Z Yao, et al. Drag reduction in turbulent flow over superhydrophobic surfaces with micro-nano textures. Mechanics in Engineering, 2013, 35(4): 20-24.

[15] S Bagherifard, R Ghelichi, M Guagliano. Numerical and experimental analysis of surface roughness generated by shot peening. Applied Surface Science, 2012, 258(18): 6831-6840.

[16] M Hajian, A Abdollah-Zadeh, S S Rezaei-Nejad. Improvement in cavitation erosion resistance of AISI 316L stainless steel by friction stir processing. Applied Surface Science, 2014, 308(3): 184-192.

[17] S K Chi, J H Park, M Y Shon. Study on cavitation erosion resistance and surface topologies of various coating materials used in shipbuilding industry. Journal of Industrial \& Engineering Chemistry, 2014, 26: 384-389.

[18] J K Choi, G L Chahine. Relationship between material pitting and cavitation field impulsive pressures. Wear, 2016, s352-353: 42-53.

[19] G L Chahine, CT Hsiao. Modelling cavitation erosion using fluid-material interaction simulations. Interface Focus A Theme Supplement of Journal of the Royal Society Interface, 2015, 5: 20150016.

[20] G L Chahine. Modelling of cavitation dynamics and interaction with material. Springer Netherlands, 2014, 106: 123-173.

[21] C T Hsiao, A Jayaprakash, A Kapahi, et al. Modelling of material pitting from cavitation bubble collapse. Journal of Fluid Mechanics, 2014, 755(9): 142-175.

[22] Giacomo Falcucci, Stefano Ubertini, Gino Bella, et al. Lattice Boltzmann simulation of cavitating flows. Commun. Comput. Phys., 2013, 49: 685-695.

[23] S S Ramesh, K M Lim, J Zheng, et al. Numerical analysis of flow induced noise propagation in supercavitation vehicles at subsonic speeds. Journal of the Acoustical Society of America, 2014, 135(4): 1752-1763.

[24] C C Tseng, W Shyy. Modeling for isothermal and cryogenic cavitations. Int J. Heat Mass Transf., 2010, 53: 513-525.

[25] K Sato, S Shimojo. Detailed observations on a starting mechanism for shedding of cavitation cloud. 5th Int. Symp. on Cavitation, Japan, November 2015

[26] G Xie, J Luo, D Guo, et al. Nanoconfined ionic liquids under electric fields. Applied Physics Letters, 2010, 96(4): 043112-043112-3.

[27] J Wu, G Wang, W Shyy. Time-dependent turbulent cavitating flow computations with interfacial transport and filter based models. International Journal for Numerical Methods in Fluids, 2005, 49(7): 739-761.

[28] M Dular, B Stoffel, B Sirok. Development of a cavitation erosion model. Wear, 2006, 261(5-6): 642-665.

[29] J P Franc, J M Michel. Fundamentals of cavitation. Kluwer Academic Publishers, 2004.

[30] B Huang, L Y Yin, G Wang, et al. Combined experimental and computational investigation of unsteady structure of sheet/cloud cavitation. Journal of Fluids Engineering, 2013, 135(7): 071301.

\section{Submit your manuscript to a SpringerOpen ${ }^{\circ}$ journal and benefit from:}

- Convenient online submission

- Rigorous peer review

- Open access: articles freely available online

- High visibility within the field

Retaining the copyright to your article

Submit your next manuscript at springeropen.com 